\title{
On the magnetic field of $\mathrm{OH} 231.8+4.2$
}

\author{
Marcelo L. Leal-Ferreira ${ }^{1}$, Wouter H. T. Vlemmings ${ }^{1,2}$, \\ Philip J. Diamond ${ }^{3}$, Athol Kemball ${ }^{4}$, Nikta Amiri ${ }^{5,6}$ \\ and Jean-François Desmurs ${ }^{7}$ \\ ${ }^{1}$ Argelander Institute für Astronomie, Universität Bonn, Germany \\ email: ferreira@astro. uni-bonn.de \\ ${ }^{2}$ OSO (Sweden), ${ }^{3} \mathrm{JBCA}$ (UK), ${ }^{4}$ University of Illinois (USA), ${ }^{5} \mathrm{JIVE}$ (Netherlands), ${ }^{6}$ Leiden \\ Observatory (Netherlands), ${ }^{7}$ OAN (Spain)
}

\begin{abstract}
During the transition from an AGB star to a planetary nebula, a large number of low/intermediate initial mass stars loses its spherical symmetry. The process responsible for that change of morphology is, so far, not well understood. The candidates responsible for shaping these objects are (i) a companion to the star (binary/heavy planet) and its tidal forces, (ii) disk interaction and (iii) magnetic fields - or a combination of these. In particular a binary induced magnetic field is a promising option. To study this we observed the polarization of $\mathrm{H}_{2} \mathrm{O}$ masers in the known binary pre-Planetary Nebula $(\mathrm{pPN}) \mathrm{OH} 231.8+4.2$. Our results show a magnetic field $B_{\|}$of $\sim 45 \mathrm{mG}$ is present in the $\mathrm{H}_{2} \mathrm{O}$ maser region of this $\mathrm{pPN}$.
\end{abstract}

Keywords. magnetic fields, masers, polarization, stars: AGB and post-AGB, stars: individual $(\mathrm{OH} 231.8+4.2)$

\section{Introduction}

The loss of spherical symmetry of evolved stars is still a matter of debate. Simulations shows that, if present, magnetic field can affect the morphology of these objects, but observations of the fields are still rare. We aim to investigate the polarization of the $\mathrm{H}_{2} \mathrm{O}$ maser emission, at $22 \mathrm{GHz}$, of the pPN OH231.8+4.2 (Rotten Egg Nebula), and infer the properties of its magnetic field. To achive that we used data from the NRAO ${ }^{\dagger}$ VLBA.

\section{Results and discussion}

We detected 30 maser features, 20 of them located in the Northern Region (NReg) and the other 10 in the Southern Region (SReg). In Fig. 1, the 30 features are scaled by their fluxes (size of the triangles), and colored according their line-of-sight velocities. The black lines, scaled in size by the amount of linear polarization, show its direction (EVPA). The origin $(0,0)$ of the plots is centered on the brightest feature we detected.

Assuming that the masers we observed correspond to roughly the same features detected by Desmurs et al. (2007), we can estimate their proper motion between the observations. We selected an area in NReg and another in SReg where the density of masers is higher and calculated how much the center point of the features have moved. The offsets we found give a velocity of the separation of $14.8 \mathrm{~km} / \mathrm{s}$ (with $d=1100 \mathrm{pc}$ and $i=36^{\circ}$; Choi et al. 2011; Kastner et al. 1992; Shure et al. 1995). Furthermore, the masers seem to be dragged on the direction of the nebula jet (Leal-Ferreira et al., in prep.). This effect can indicate that the masers arise in a turbulent material that is entrained by the jet.

Linear polarization was found for 3 of the 11 maser features in the SReg (see Fig. 1). A high scatter is present in the EVPA of these features and we can point out two different 

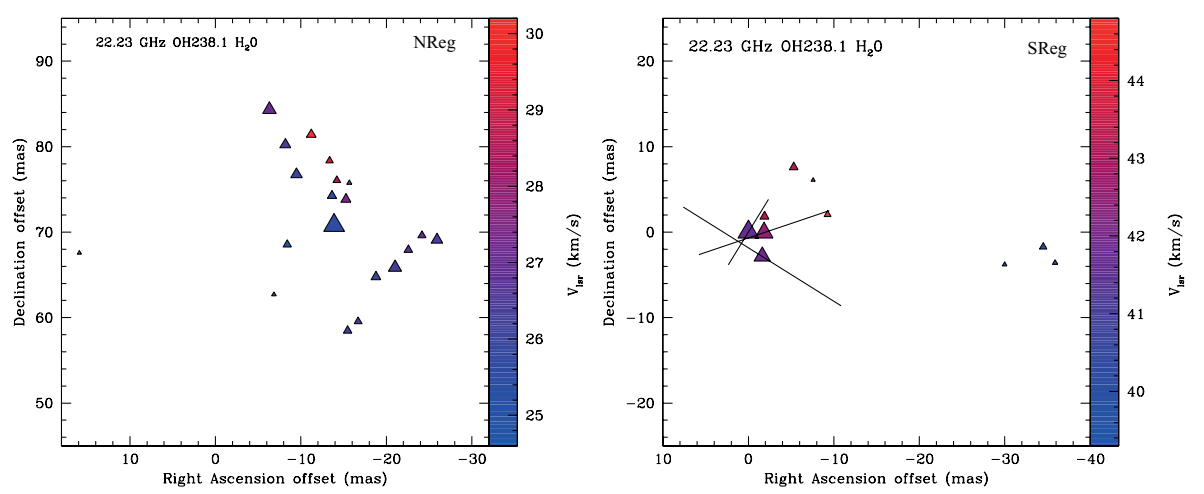

Figure 1. $\mathrm{H}_{2} \mathrm{O}$ maser features of $\mathrm{OH} 231.8$. The triangles are scaled in size by the maser fluxes, and in color by their velocities. The lines show the EVPA and intensity of the linear polarization.
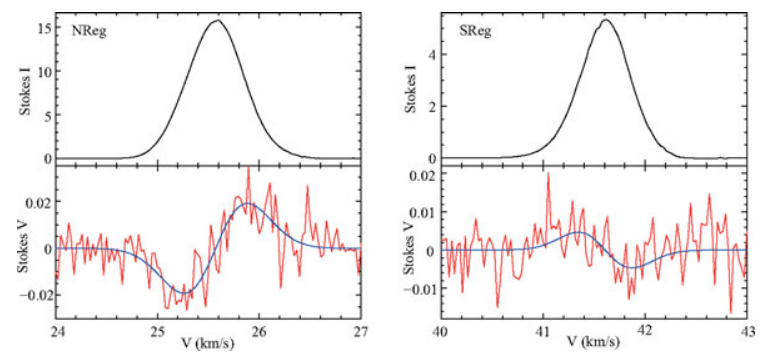

Figure 2. Spectra of the Stokes $I$ and $V$. The blue lines show the model fits.

scenarios to interpret it: (i) The scatter could be caused by turbulence, or (ii) in case of a toroidal magnetic field, the masers could be located on its tangent points.

We also detected circular polarization on the 2 strongest maser features we observed. Figure 2 shows the spectra of Stokes $I$ and $V$. Overplotted to the $V$ spectrum (red), we show the model curve that fits the spectrum (blue). From this fit, we found that the strength of the magnetic field is $B_{\|}(\mathrm{NReg})=44 \pm 7 \mathrm{mG}$ and $B_{\|}(\mathrm{SReg})=-29 \pm 21 \mathrm{mG}$.

The magnetic field morphology is still not defined, as different shapes can fit our polarization results. However, assuming a toroidal field $(B \propto 1 / r)$ and a distance of $\sim 40$ AU between the central star and each maser region, it is possible to project the field strength on the stellar surface (taken to be $\sim 1 \mathrm{AU}$ ). We then found $\sim 1.5 \mathrm{G}<B_{\text {surface }}<$ $\sim 2 \mathrm{G}$, similar to that estimated for the pPN W43A (Vlemmings et al. 2006).

These results, allied with the ones from Etoka et al. (2009), make OH231.8 the first evolved star, known to be a binary, in which the presence of a magnetic field is confirmed.

\section{References}

Choi, Y. K., Brunthaler, A., Menten, K. M., \& Reid, M. J. 2011, this conference proceedings Desmurs, J. F., Alcolea, J., Bujarrabal, V., et al. 2007, A\& A, 468, 189

Etoka, S., Zijlstra, A., Richards, A. M., Matsuura, M., \& Lagadec, E. 2009, in: B. Soonthornthum, S. Komonjinda, K. S. Cheng, and K. C. Leung (eds.), The Eighth Pacific Rim Conference on Stellar Astrophysics: A Tribute to Kam-Ching Leung, (San Francisco: Astronomical Society of the Pacific), 404, 311

Leal-Ferreira, M. L., Vlemmings, W. H. T., Diamond, P. J., Kemball, A., et al. 2011, A\&A, in prep.

Kastner, J. H., Weintraub, D. A., Zuckerman, B., Becklin, E. E., et al. 1992, ApJ, 398, 552

Shure, M., Sellgren, K., Jones, T. J., \& Klebe, D. 1995, AJ, 109, 721

Vlemmings, W. H. T., Diamond, P. J., \& Imai, H. 2006, Nature, 440, 58 